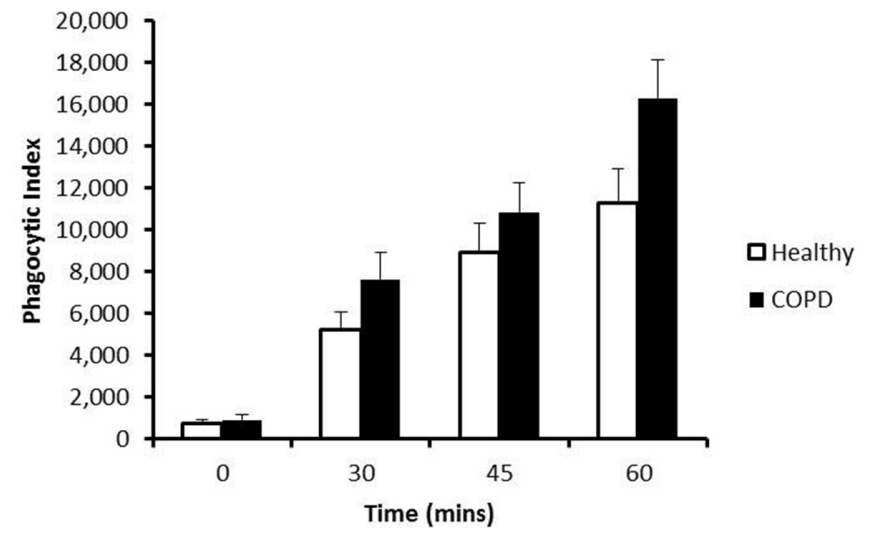

Abstract S46 Figure 1 Phagocytosis of unopsonised SA by blood neutrophils over $60 \mathrm{~min}$

20), or Escherichia coli bioparticles (EC, $\mathrm{n}=10$ ) and fluorescently labelled disease-relevant bacteria, Haemophilus influenzae (HI, $\mathrm{n}$ $=10)$ and Streptococcus pneumoniae (SP, $\mathrm{n}=10$ ) was assessed, at regular intervals over $60 \mathrm{~min}$, using flow-cytometry. Results were confirmed using time-lapse video microscopy.

Results Peak phagocytosis was achieved at $60 \mathrm{~min}$ for unopsonised bacteria and $30 \mathrm{~min}$ for opsonised bacteria. There were no differences in time to peak phagocytosis between bacterial species. Blood neutrophils from patients with COPD and HC displayed similar phagocytic ability, in both percentage of neutrophils with phagocytic activity and the amount of SA, EC, HI or SP ingested (as indicated by MFI) (COPD vs. HC, p > 0.05 for all). This was ubiquitous to both opsonin independent and opsonin-dependant phagocytosis, and was consistent across all time points measured. A typical comparison is shown in figure one, with unopsonised SA data.

Conclusions Phagocytic ability of blood neutrophils from patients with COPD to ingest Staphylococcus aureus, Escherichia coli, Streptococcus pneumoniae and Haemophilus influenzae is not altered compared to age-matched healthy controls. This should be replicated in lung neutrophils to assess whether transmigration to the tissues affects function.

\section{S47 ENHANCED IL-6/CCL3 SIGNALLING IN THE PLASMA OF PATIENTS WITH COPD}

${ }^{1}$ AK Ravi, 'S Khurana, ${ }^{1} \mathrm{~A}$ Banyard, 'J Plumb, 'G Booth, ${ }^{2} \mathrm{M}$ Catley, ${ }^{2} \mathrm{~L}$ Healy, ${ }^{2} \mathrm{E}$ Smith, IJ Vestbo, 'D Singh. 'The University of Manchester, Manchester Academic Health Science Centre, University Hospital of South Manchester NHS Foundation Trust, NIHR South Manchester Respiratory and Allergy Clinical Research Facility, Manchester, UK; ${ }^{2} U C B$, Slough, UK

\subsection{6/thoraxjnl-2014-206260.53}

Rationale IL-6 is a pro-inflammatory cytokine that signals through soluble (sIL-6R/sgp80) and membrane bound (gp80) receptors to promote recruitment of mononuclear cells. IL-6 induces expression of CCL3, a monocytic chemokine. Monocytes are precursors of macrophages and dendritic cells. They can be classified into three subtypes according to surface expression of CD14 (LPS receptor) and CD16 (FcgammaRIII): CD14++CD16-, CD14+CD16+, CD14-CD16++. We measured plasma levels of IL-6, sIL-6R and CCL3 and determined the chemokine receptor expression profile of circulating monocytes in COPD.

Methods 70 COPD patients and 30 healthy controls comprising 15 smokers (S) and 15 healthy non-smokers (HNS) underwent plasma sampling. Levels of IL-6, sIL-6R and CCL3 were
Abstract S47 Table 1

\begin{tabular}{lllll}
\hline & COPD & S & HNS & ANOVA \\
\hline IL-6 $(\mathrm{pg} / \mathrm{ml})$ & $33.7(190.3)$ & $3.8(1.9)$ & $0^{*}$ & $\mathrm{p}<0.0001$ \\
sIL-6R (pg/ml) & $5338(850.3)$ & $4453(613.2)$ & $4853(856.8)$ & $\mathrm{p}=0.0005$ \\
CCL3 $(\mathrm{pg} / \mathrm{ml})$ & $74.8(111.9)^{* *}$ & $0^{*}$ & $0^{*}$ & - \\
\hline
\end{tabular}

Data expressed as mean (SD) * < lower limit of quantification of the assay ${ }^{*} \mathrm{CCL} 3$ levels registered above the assay's lower limit of quantification in 7/70 COPD patients.

determined by multiplex analysis (MSD) of plasma. Multi-colour flow cytometry was performed on whole blood obtained from 32 COPD patients, $8 \mathrm{~S}$ and $8 \mathrm{HNS}$ to measure surface expression levels of chemokine receptors CCR1, CCR2, CCR7, CXCR1 and CX3CR1 on CD14++CD16-, CD14+CD16+ and CD14-CD16++ monocytes.

Results COPD patients had the greatest levels of IL-6 and sIL6R. CCL3 was not detected in any controls, but was present in a subset of COPD patients.\% surface expression of the CCL3 receptor CCR1 measured on CD14++CD16- monocytes of COPD patients was greater than those of HNS $(p=0.04)$. There were no significant differences in expression levels of other chemokine receptors.

Conclusion We report evidence of enhanced IL-6 signalling in the plasma of COPD patients and increased plasma CCL3 in a subset of individuals from this disease group. Furthermore, there was increased CCR1 expression on COPD monocytes. Enhanced IL-6 may co-ordinate the mononuclear component of the inflammatory response in COPD.

\section{S48 AIR POLLUTION PARTICULATE MATTER PROMOTES DC MATURATION AND ENHANCES THEIR STIMULATION OF CD8 LYMPHOCYTE RESPONSES}

TR Ho, PE Pfeffer, E Mann, FJ Kelly, NC Matthews, CM Hawrylowiz. King's College London, London, UK

\subsection{6/thoraxjnl-2014-206260.54}

Background High levels of ambient urban particulate matter (UPM), a component of air pollution, are associated with respiratory tract infections and exacerbations of airways diseases. Dendritic cells (DCs) exposed to inhaled UPM orchestrate the resulting immune response. We have previously shown that UPM-stimulation of DCs results in enhanced proliferation of naïve CD4 lymphocytes but decreased priming of IFN $\gamma$-producing CD4 lymphocytes. These CD4 lymphocytes are important in anti-viral immune responses; however, Tc1 CD8 lymphocytes have more direct anti-viral action. In this research we have studied the effect of UPM on DC priming of CD8 lymphocytes. Methods CD1c peripheral blood DCs were isolated, cultured in the presence/absence of UPM stimulation, with GM-CSF or in medium alone. DC expression of CD83, CCR7, CD40 and MHC Class I were measured by flow-cytometry at $24 \mathrm{~h}$. Pretreated DCs were also cultured with naïve CD8 lymphocytes in

Abstract S48 Table 1 Effect of UPM stimulation of DCs upon naïve CD8 lymphocyte response in MLR at day 5. Median (Inter-Quartile Range) TNF $\alpha$, IFN $\gamma$ and IL-13 production

\begin{tabular}{lllll}
\hline & Control & UPM & GM-CSF & UPM + GM-CSF \\
\hline TNF $\alpha$ (pg/ml) & $77.6(60.2-256)$ & $149(72.3-853)$ & $904(148-1425)$ & $2411(434-4869)$ \\
IFN $\gamma(\mathrm{pg} / \mathrm{ml})$ & $84.5(44.9-195)$ & $225(73.6-1537)$ & $1009(66.25-1477)$ & $2343(189-8726)$ \\
$\mathrm{IL}-13(\mathrm{pg} / \mathrm{ml})$ & $25.9(5.88-106)$ & $59.0(25.3-335)$ & $939(45.8-984)$ & $638(74.6-1266)$ \\
\hline
\end{tabular}

